\title{
D
}

Pulmonary Medicine, Salmaniya Medical Complex, Manama, Bahrain.

\section{An interesting case of undiagnosed pleural effusion}

\section{Case report}

Pleural effusions are commonly encountered in the clinical practise of both respiratory and nonrespiratory specialists. An estimated 1-1.5 million new cases in the USA and 200000-250000 new cases of pleural effusions are reported from the UK each year [1]. Analysis of the relevant clinical history, physical examination, chest radiography and diagnostic thoracentesis is useful in identifying the cause of pleural effusion in majority of the cases [2]. In a few cases, the aetiology may be unclear after the initial assessment. The list of diseases that may account for a persistent undiagnosed pleural effusion is long [3]. We present an interesting case of undiagnosed pleural effusion that was encountered in our hospital.

\section{Case presentation}

A 33-year-old male presented to our hospital with a history of sudden-onset, pleuritic, right-sided chest pain of 2 days' duration. It was not associated with fever, cough, dyspnoea, wheeze or haemoptysis. There was no history of swelling in the lower extremities, weight loss or anorexia. There was no history of any significant comorbidities. He was a smoker (8 packyears). He had no history of drug abuse.

On examination, his temperature was $37.2^{\circ} \mathrm{C}$, pulse was 77 beats. $\mathrm{min}^{-1}$, respiratory rate was 22 breaths. $\mathrm{min}^{-1}$, blood pressure was 112/66 mmHg and oxygen saturation measured by pulse oximetry was $98 \%$ on room air. There was no pallor, clubbing, pedal oedema, icterus or lymphadenopathy. The breath sounds were absent in the infrascapular and infra-axillary areas on the right side. No adventitious sounds were heard on either side of the chest. The remaining systemic examination was unremarkable.
Investigations revealed a haemoglobin level of $16.4 \mathrm{~g} \cdot \mathrm{dL}^{-1}$, and total leukocyte count of 8870 cells $\cdot \mathrm{mm}^{-3}$ with a differential count of $62 \%$ neutrophils, $28 \%$ lymphocytes, $7 \%$ monocytes, $2 \%$ eosinophils and $1 \%$ basophils. The platelet count was 160000 cells. $\mathrm{mm}^{-3}$. Creatinine, electrolytes and liver function tests were normal. The ECG was unremarkable and cardiac enzymes were within normal limits. Chest radiograph (figure 1) showed a mild, right-sided pleural effusion, blunting of the left costophrenic angle, no shift of mediastinal position and no lung parenchymal opacities.

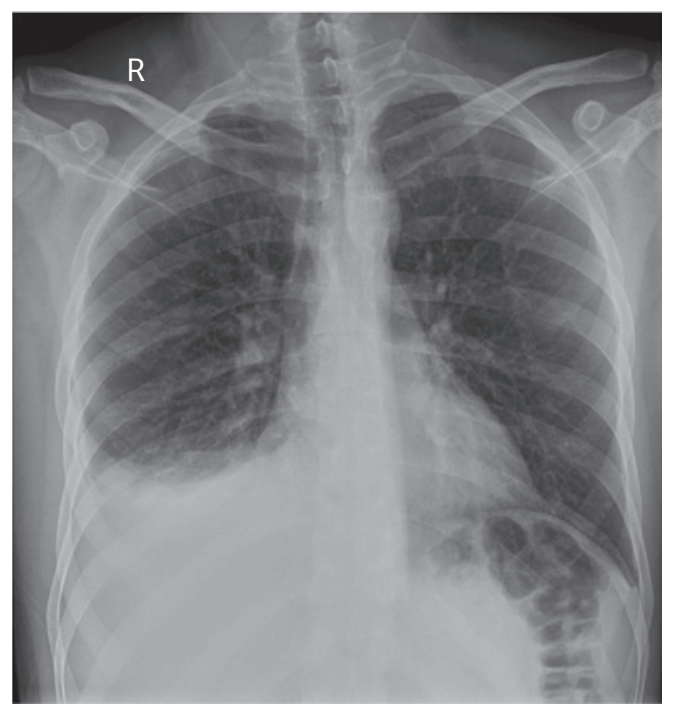

Figure 1 Frontal chest radiograph.

Task 1

What is the next step in management?
Cite as: Panjwani A, Zaid T. An interesting case of undiagnosed pleural effusion. Breathe 2017; 13: e46-e52. 


\section{Answer 1}

Bilateral effusions that are of unequal size, unresponsive to therapy and associated with pleuritic chest pain or fever should be subjected to diagnostic thoracentesis [4].

He was subjected to a diagnostic thoracentesis, which showed $6.5 \mathrm{~g} \cdot \mathrm{L}^{-1}$ protein (corresponding serum proteins were $7.3 \mathrm{~g} \cdot \mathrm{L}^{-1}$ ), and lactate dehydrogenase (LDH) concentration was $820 \mathrm{U} \cdot \mathrm{L}^{-1}$ (corresponding serum LDH was $563 \mathrm{U} \cdot \mathrm{L}^{-1}$ ) and that of glucose was $7 \mathrm{mmol} \cdot \mathrm{L}^{-1}$. The erythrocyte count was 2700000 cells. $\mu \mathrm{L}^{-1}$, and leukocytes count was 3400 cells. $\mu L^{-1}$ with $53 \%$ neutrophils,
$42 \%$ lymphocytes and 5\% monocytes. The Gram stain and cultures were sterile. There were no acid-fast bacilli seen in smears and real-time PCR for Mycobacterium tuberculosis was negative. The cytological examination showed no malignant cells.

He was treated with antibiotics and analgesics. $48 \mathrm{~h}$ after admission, the patient complained of severe, left-sided chest pain in the night. It was nonradiating and not associated with sweating, nausea or vomiting. There was no haemodynamic compromise and the patient was normoxaemic. There were reduced breath sounds in both infrascapular and infra-axillary areas. An ECG was performed (figure 2).

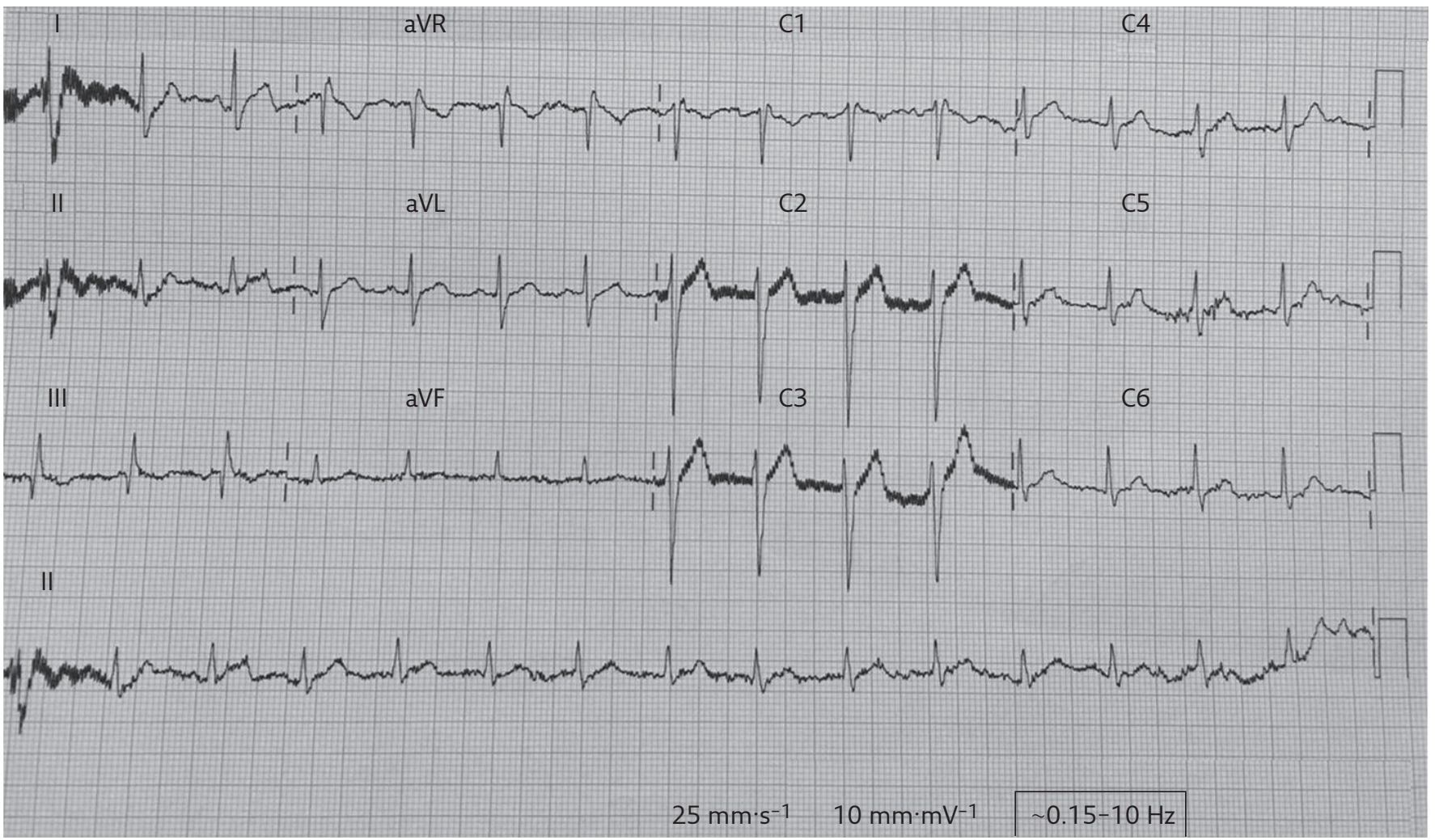

Figure $2 E C G$.

Task 2

1) Describe the ECG findings.

2) What is the differential diagnosis for these findings? 


\section{Answer 2}

1) The ECG showed a normal sinus rhythm with an S-wave in lead I, and a Q-wave and an inverted T-wave in lead III. This pattern is the S1Q3T3 pattern. There was an inverted $\mathrm{T}$-wave in V1.

2) The S1Q3T3 pattern is a sign of acute cor pulmonale. The differential diagnosis for this condition includes acute pulmonary embolism (PE), pneumothorax, acute bronchospasm and left posterior fascicular block. Severe pneumonia may also show this pattern in almost $10 \%$ of cases [5]. It is seen in $12-50 \%$ of cases of acute PE [6]. This pattern has $54 \%$ sensitivity, $62 \%$ specificity, $80 \%$ positive predictive value and $33 \%$ negative predictive value for the diagnosis of acute PE.

Serial cardiac enzymes were normal. Chest radiography was also repeated (figure 3 ).

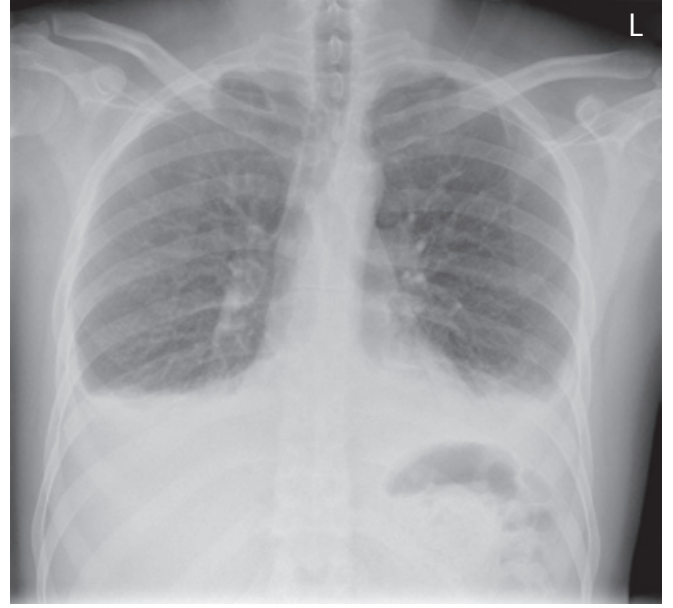

Figure 3 Frontal chest radiograph.

Task 3

Describe the chest radiograph. 


\section{Answer 3}

The second chest radiograph showed an increase in the pleural effusion on the left side, while the right-sided effusion was the same. There were no fresh lung parenchymal opacities.

The patient had a bout of cough associated with mild haemoptysis and fever of up to $38^{\circ} \mathrm{C}$. The possibility of PE was entertained. The revised Geneva score for the assessment of the clinical probability of PE was 5, suggesting that there was an intermediate probability of an embolism.
Therefore, a D-dimer test was ordered and the level was found to be $3.6 \mathrm{mg} \cdot \mathrm{L}^{-1}$ (normal range 0.09-0.33 $\left.\mathrm{mg} \cdot \mathrm{L}^{-1}\right)$. In line with the diagnostic algorithm for patients with suspected, not high-risk PE proposed by the European Society of Cardiology [7], the patient was subjected to computed tomographic pulmonary angiography (CTPA), which showed filling defects in the both lower and upper lobar arteries, right middle lobe, and lingular arterial branches consistent with an acute PE. There was no right ventricle enlargement seen in the fourchamber view of the heart by CTPA, which was suggestive that there was no right ventricular dysfunction (figure 4).
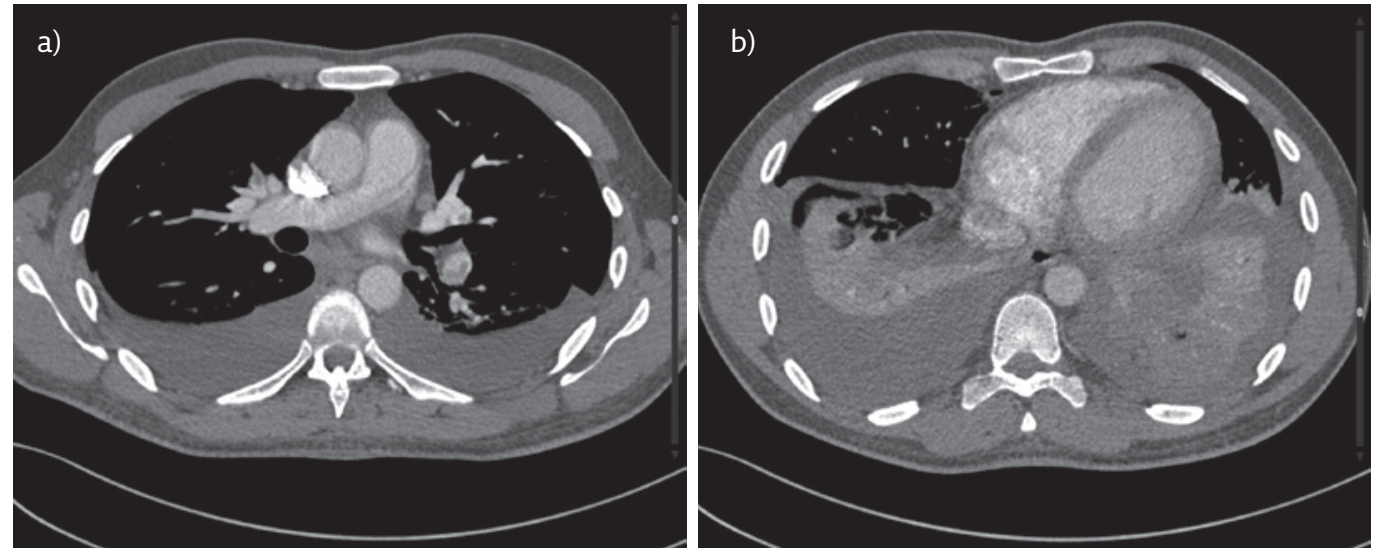

Figure 4 a) CTPA showing filling defects in both the upper and left lower lobar pulmonary arteries suggestive of bilateral pulmonary embolism. Bilateral, mild pleural effusion is also seen. b) CTPA showing no enlargement of the right ventricle with a normal position of the interventricular septum suggesting no evidence of right ventricle dysfunction.

Task 4

How would you manage this patient? 


\section{Answer 4}

Once a diagnosis of acute PE is confirmed, further optimal management depends on stratifying the patient into classes of disease severity in such a way that the initial treatment is planned according to the individual's early death risk. The clinical tools used to stratify patients into high, intermediate and low risk of early mortality are the presence of shock or hypotension, Pulmonary Embolism Severity Index (PESI) and imaging, as well as laboratory markers of right ventricular dysfunction. PESI classes I-II represent low-risk patients who may be managed with anticoagulants and considered for early discharge from the hospital. If there is any doubt about the presence of right ventricular dysfunction even in patients belonging to PESI class I-II, further imaging and laboratory testing of right ventricular function should be done [8]. PESI class III-IV patients with normal blood pressure, and imaging and/or laboratory evidence of right ventricular dysfunction need to be put on anticoagulation and monitored for haemodynamic parameters. Primary reperfusion therapy should be considered in these patients when clinical signs of a haemodynamic compromise appear.

The patient was found to be in PESI class I. This, combined with there being no signs of right ventricular dysfunction on CTPA and normal troponin levels, was suggestive of a low risk of adverse early outcome. He was given enoxaparin 7 days, followed by dabigatran. Blood and sputum cultures were eventually found to be sterile. The autoimmune/vasculitis screen was negative. Gradually, over the next few days, the patient's fever subsided and his chest pain resolved completely. He was continued on dabigatran and a repeat chest radiograph at the last follow-up showed a complete resolution of the pleural effusion on left side with a residual blunting of right costophrenic angle (figure 5). A pleural fluid acid-fast bacillus culture showed no growth of any organism after 6 weeks of incubation.

\section{Discussion}

The evaluation of a persistent, undiagnosed pleural effusion begins with the classification of the pleural fluid as a transudative or exudative effusion. Light's criteria are the best way to differentiate transudates from exudates. In approximately $15-30 \%$ cases, transudates are misclassified as exudates. These false-positive exudates are usually seen in patients receiving diuretics. Analysis of protein and albumin gradients in these patients can accurately detect transudative nature of the pleural effusion [9]. Malignancy, pneumonia,

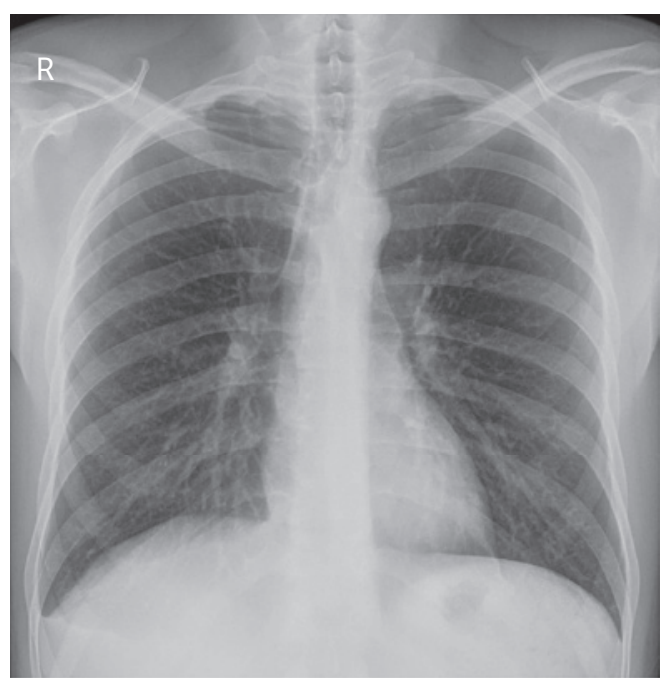

Figure 5 Frontal chest radiograph.

tuberculosis, PE, fungal infections, pancreatic pseudocysts and intra-abdominal abscess are some of the diseases likely to produce exudative pleural effusions [3]. Special tests on the pleural fluid help in identifying the cause of the pleural effusion in many cases. Measurement of adenosine deaminase or $\gamma$-interferon helps in the diagnosis of pleural tuberculosis. If a lymphoma is suspected, flow cytometry helps in establishing the diagnosis [10]. In cases of suspected chylothorax, pleural fluid cholesterol and triglycerides help in confirming the diagnosis [4]. Spiral computed tomography of the chest is recommended in the evaluation of undiagnosed pleural effusions. Parenchymal infiltrates and masses, pleural thickening and masses, mediastinal lymphadenopathy, and PE can be diagnosed with these scans. If the diagnosis is still elusive, thoracoscopy and pleural biopsy are recommended [11].

Acute PE is the third most frequent cardiovascular syndrome after myocardial infarction and stroke [8]. It is a cause of significant acute and long-term morbidity and mortality worldwide. Approximately one-third of the cases of PE die in the first 30 days. Early diagnosis and appropriate treatment can reduce the mortality to $<10 \%$. Pleural effusions occur in 19-61\% of cases of PE [12]. Most of the series of pleural effusions that have been investigated with thoracentesis have $<5 \%$ of cases attributable to $P E$. In a recent publication, $1.8 \%$ of the cases of pleural effusion were caused by PE [13]. The possible reasons for this discrepancy are [14]:

- Most of the effusions related to PE are too small to warrant a thoracentesis

- It is not considered as a possibility while investigating cases of undiagnosed pleural effusion

- Most of the patients with moderate to high suspicion of PE are immediately anticoagulated by the time the diagnosis is established and thoracentesis is not indicated in such a situation 
$P E$ is the most overlooked entity in patients with pleural effusion. A substantial number of cases of undiagnosed pleural effusions may be caused by acute PE.

The release of vascular endothelial growth factor and other inflammatory mediators causes an increase in the pulmonary capillary permeability. The resultant fluid in the interstitial space traverses the visceral pleura and accumulates in the pleural cavity, causing an exudative pleural effusion. This is the most plausible explanation for the presence of pleural effusion in patients with acute PE. There is long list of risk factors for a patient developing acute PE. These include a recent history of surgery, previous venous thromboembolism, immobilisation due to any cause, trauma, malignancy, and hereditary and acquired thrombophilia, such as prothrombin G20210A mutation or activated protein $C$ resistance. Advanced age, obesity, pregnancy, the post partum period, central venous catheters and acute infections are some of the other factors implicated in the causation of acute PE. Onefifth of the cases of acute PE have no identifiable risk for its occurrence.

PE may present as pulmonary infarction with pleuritic chest pain and/or haemoptysis, isolated dyspnoea or circulatory collapse. In a large study of 4145 patients with $\mathrm{PE}$, pleural effusion was found in $27 \%$ of cases with pulmonary infarction, $16 \%$ cases with circulatory collapse and $12 \%$ cases with isolated dyspnoea [15]. Pleuritic chest pain is the most important symptom in patients presenting with $\mathrm{PE}$ and pleural effusion, occurring in more than three-quarters of the cases. Thus, the presence of pleuritic chest pain in a patient with pleural effusion should raise a strong suspicion of acute PE. Dyspnoea is the other common symptom, seen in almost $70 \%$ of cases of pleural effusion secondary to acute PE. It is usually out of proportion to the size of the pleural effusion. Cough and fever may be seen in half the cases of pleural effusion with PE. $15 \%$ of the cases may have haemoptysis [14]. Our patient had pleuritic chest pain, haemoptysis (suggesting pulmonary infarction), fever and cough.

Pleural effusion associated with PE are usually maximal by the third day and any enlargement of effusion after this period or contralateral occurrence of effusion is suggestive of recurrent embolism [12], as was seen in our case. The pleural effusion is small and unilateral in majority of the patients, but it maybe bilateral in one-third of patients [16]. In a study of 141 patients with unilateral pleural effusion subjected to CTPA after a comprehensive clinical assessment including a diagnostic thoracentesis, $6.4 \%$ of cases were found to have PE. Almost all these cases were found to have cytologically proven malignant pleural effusion. PE was not found to be a primary cause of effusion in these cases. The authors concluded that pulmonary emboli are not a common cause of unilateral pleural effusion but may be frequently seen in patients having a concomitant pleural malignancy. They advocated the use of CTPA and pleural-phase imaging for patients presenting with a pleural effusion and suspected pleural malignancy [17]. Up to one-third of the cases may have loculated effusions. This is usually a result of a delay in the diagnosis of the PE. Systemic anticoagulants may be helpful in the resolution of these loculated collections [18].

Pleural fluid analysis reveals exudative fluid, which shows an increase in the erythrocyte count $>10000$ cells $\mu \mathrm{L}^{-1}$ in most of the cases [19]. There may be an increase in the leukocyte count and neutrophilic preponderance. A few patients may also have pleural fluid eosinophilia. Our patient had an exudative effusion with increased erythrocyte and leukocyte counts, and predominant neutrophils in the pleural fluid.

The treatment of pleural effusion associated with $P E$ is similar to that of any other patient of PE, and depends on risk stratification and consequent further management. Anticoagulation is the mainstay of therapy in all cases. The presence of haemorrhagic effusion is not a contraindication for anticoagulation or thrombolysis [12]. The pleural effusion gradually resolves with anticoagulant therapy, as was seen in our case. If the pleural effusion increases in size or appears on the contralateral side, it is suggestive of a recurrent thromboembolism, infection in the pleural cavity or haemothorax [14].

\section{Conclusion}

Pleural effusions are commonly seen in association with acute PE. It should be entertained as an important possibility while investigating a case of undiagnosed exudative pleural effusion presenting with a pleuritic chest pain. These effusions are generally unilateral but may also be bilateral especially if there is a recurrent thromboembolism. The pleural effusion associated with acute PE show a good response to systemic anticoagulant therapy.

\section{Conflict of interest}

None declared

\section{References}

1. Bhatnagar R, Maskell $\mathrm{N}$. The modern diagnosis and management of pleural effusions. BMJ 2015; 351: h4520.
2. Porcel JM. Pearls and myths in pleural fluid analysis. Respirology 2011; 16: 44-52. 
3. Light RW. The undiagnosed pleural effusion. Clin Chest Med 2006; 27: 309-319.

4. McGrath EE, Anderson PB. Diagnosis of pleural effusion: A systematic approach. Am J Crit Care 2011; 20: 119-127.

5. Seedat MA, Feldman C, Skoularigis J, et al. A study of acute community-acquired pneumonia, including details of cardiac changes. Q J Med 1993; 86: 669-675.

6. Ferrari E, Imbert A, Chevalier T, et al. The ECG in pulmonary embolism. Predictive value of negative T waves in precordial leads - 80 case reports. Chest 1997; 111: 537-543.

7. Konstantinides SV, Torbicki A, Agnelli G, et al. 2014 ESC guidelines on the diagnosis and management of acute pulmonary embolism. Eur Heart J 2014; 35: 3033-3069.

8. Barco S, Konstantinides S. Pulmonary embolism. In: Heunks L, Demoule A, Windisch W, eds. Pulmonary Emergencies (ERS Monograph). Sheffield, European Respiratory Society, 2016; pp. 15-31.

9. Romero-Candeira S, Hernández L, Romero-Brufao S, et al. Is it meaningful to use biochemical parameters to discriminate between transudative and exudative pleural effusions? Chest 2002; 122: 1524-1529.

10. Alexandrakis MG, Passam FH, Kyriakou DS, et al. Pleural effusions in hematologic malignancies. Chest 2004; 125: 1546-1555.

11. Nattusamy L, Madan K, Mohan A, et al. Utility of semirigid thoracoscopy in undiagnosed exudative pleural effusion. Lung India 2015; 32: 119-126.
12. Agarwal R, Singh N, Gupta D. Pleural effusions associated with pulmonary thromboembolism: A systematic review. Indian J Chest Dis Allied Sci 2009; 51: 159-164.

13. Bintcliffe OJ, Lee GY, Rahman NM, et al. The management of benign non-infective pleural effusions. Eur Respir Rev 2016; 25: 303-316.

14. Light RW. Pleural effusion in pulmonary embolism. Semin Respir Crit Care Med 2010; 31: 716-722.

15. Lobo JL, Zorrilla V, Aizpuru F, et al. Clinical syndromes and clinical outcome in patients with pulmonary embolism: findings from the RIETE registry. Chest 2006; 130: 1817-1822.

16. Liu M, Cui A, Zhai ZG, et al. Incidence of pleural effusion in patients with pulmonary embolism. Chin Med J 2015; 128 : 1032-1036.

17. Hooper C, Laurence I, Harvey J, et al. The role of CT pulmonary angiography in the investigation of unilateral pleural effusions. Respiration 2014; 87: 26-31.

18. Erkan L, Fýndýk $\mathrm{S}$, Uzun $\mathrm{O}$, et al. A new radiologic appearance of pulmonary thromboembolism: Multiloculated pleural effusions. Chest 2004; 126: 298-302.

19. Romero Candeira S, Hernández Blasco L, Soler MJ, et al. Biochemical and cytologic characteristics of pleural effusions secondary to pulmonary embolism. Chest 2002; 121: 465-469. 